The main contribution in second integral term of (B1) gives the $\left(\omega_{k}-\omega\right)$ frequencies. If $\left\langle q_{k}^{02}\right\rangle$ varies slowly near $\omega$ we have

$$
\begin{aligned}
\left\langle\left\langle x(t) x\left(t^{\prime}\right)\right\rangle\right\rangle_{V} & =\left(k T / \omega^{2}\right) \cos \omega\left(t-t^{\prime}\right) \exp \left[-\frac{1}{2} \eta\left(t+t^{\prime}\right)\right] \\
& +\frac{k T}{\omega^{2}} \frac{\alpha^{2}}{\omega^{2}} \exp \left[-\frac{1}{2} \eta\left(t+t^{\prime}\right)\right] \sum_{k} \int_{0}^{t} d t_{1} \int_{0}^{t^{\prime}} d t_{2} \exp \left[\frac{1}{2} \eta\left(t_{1}+t_{2}\right)\right] \sin \omega\left(t-t_{1}\right) \sin \omega\left(t^{\prime}-t_{2}\right) \cos \omega_{k}\left(t_{1}-t_{2}\right) .
\end{aligned}
$$

Bearing in mind the condition $\omega_{D} \tau$ we find

$$
\left\langle\left\langle x(t) x\left(t^{\prime}\right)\right\rangle\right\rangle_{V}=\frac{k T}{\omega^{2}} \cos \omega\left(t-t^{\prime}\right) \exp \left[-\frac{1}{2} \eta\left(t+t^{\prime}\right)\right]-\frac{k T}{\omega^{2}} \eta \exp \left[-\frac{1}{2} \eta\left(t+t^{\prime}\right)\right] \int_{0}^{t^{\prime}} e^{n t_{1}} \sin \omega\left(t-t_{1}\right) \sin \omega\left(t^{\prime}-t_{1}\right) d t_{1} .
$$

Here the integration may be easily done. So we get the result (3.16).

THE JOURNAL OF CHEMICAL PHYSICS

VOLUME 47 , NUMBER $10 \quad 15$ NOVEMBER 1967

\title{
Molecular Beam Measurement of the Hyperfine Structure of ${ }^{133} \mathrm{Cs}^{19} \mathrm{~F}^{*} \dagger$
}

\author{
Thomas C. English $\ddagger$ ANd Jens C. Zorn \\ Randall Laboratory of Physics, The University of Michigan, Ann Arbor, Michigan
}

(Received 29 June 1967)

\begin{abstract}
The magnitudes and signs of the hyperfine interaction constants for ${ }^{133} \mathrm{Cs}^{19} \mathrm{~F}$ in the $J=1, v=0,1,2$ states have been measured with a molecular beam electric resonance spectrometer. The results for $v=0$ are: $e Q_{1} q_{1}=1.2370(13) \mathrm{MHz}, c_{1}=0.70(7) \mathrm{kHz}, c_{2}=15.1(6) \mathrm{kHz}, c_{3}=0.92(12) \mathrm{kHz}, c_{4}=0.61(10) \mathrm{kHz}$. The numbers in parentheses are uncertainties in units of the last digit given. $e Q_{1} q_{1}$ is the quadrupole coupling constant for the cesium nucleus, $c_{1}$ and $c_{2}$ are the spin-rotation coupling constants for the cesium and fluorine nuclei, respectively, and $c_{3}$ and $c_{4}$ are the coupling constants for the tensor and scalar parts of the nuclear spin-spin interaction. These constants are obtained from the radio-frequency spectrum of CsF taken under veryweak-field conditions $\left(E_{\mathrm{do}}=1.5 \mathrm{~V} / \mathrm{cm} ; H<0.05 \mathrm{Oe}\right)$; some previously ignored contributions of the fluorine spin-rotation interaction and the spin-spin interaction are clearly evident. The observed variation in $e Q_{1} q_{1}$ with vibrational state shows only qualitative agreement with theory. An appendix gives compact expressions for the very-weak-field hfs energy levels of a $1 \Sigma$ molecule in which only one nucleus has a quadrupole moment.
\end{abstract}

\section{INTRODUCTION}

The present experiment on $\mathrm{CsF}$ was undertaken as a part of a program in which the resolution capabilities of modern molecular beam electric resonance (MBER) spectrometers are utilized for the detailed study of electric and magnetic interactions within diatomic molecules. The magnitudes of these interactions and their variation with quantum state are of interest to many workers; we are particularly concerned with a detailed understanding of the magnetic-dipole and electric-quadrupole interactions (which are the only ones that are expected to contribute to the hfs of the $J=1$ rotational state) in order that the more complex radio-frequency spectrum of $J>2$ states may be closely examined for evidence of new interactions, for example, that of a nuclear electric hexadecapole moment. Aside from giving precise values of the hfs constants of $\mathrm{CsF}$, this work shows that the analysis of high-resolution

${ }^{*}$ Research supported in part by the U.S. Atomic Energy Commission.

$\dagger$ Submitted by T. C. English in partial fulfillment of the requirements for the Ph.D. degree in physics at the University of Michigan.

$\ddagger$ NASA Predoctoral Fellow, 1963-1966. Present address: Physics Department, Harvard University, Cambridge, Mass.
MBER spectra requires the consideration of contributions to the magnetic-dipole interaction energies which have heretofore been neglected in very-weak-field electric-resonance experiments; these contributions will be important if any new interactions are to be unambiguously identified in $J \geq 2$ spectra.

The pioneering MBER work was done on the CsF molecule. ${ }^{1,2}$ The results provided the stimulus for measurements on other alkali halides and also for a number of theoretical investigations of interactions in heavy molecules. More recently, a substantial amount of information about electron-coupled nuclear spin-spin interactions $^{3}$ (which were originally discovered in highresolution NMR experiments) has been provided by MBER measurements. At the time our experiment was undertaken the electron-coupled interactions in $\mathrm{CsF}$ had not been studied; moreover the values of the hfs constants for CsF determined in the early MBER work were still the most accurate available, notwithstanding subsequent experiments by the methods of molecular-

\footnotetext{
${ }^{1}$ H. K. Hughes, Phys. Rev. 72,614 (1947).

${ }^{2}$ J. W. Trischka, Phys. Rev. 74, 718 (1948); 76, 1365 (1949).

${ }^{3}$ N. F. Ramsey, Molecular Beams (Oxford University Press, London, 1956), p. 206, 231.
} 
beam magnetic resonance $e^{4,5}$ and microwave absorption spectroscopy. ${ }^{6}$ While the present experiment was in progress, measurements on the hfs of CsF done with a combined Stark-Zeeman method of electric resonance were reported by Gräff and Runólfsson ${ }^{7}$; their experiment was performed in strong external $E$ and $H$ fields and is, therefore, complementary to our very-weak-field experiment.

The details of construction of the MBER apparatus used in this experiment are given elsewhere ${ }^{8}$; the signal strengths and other conditions of operation are similar to those described in our paper ${ }^{9}$ on $\mathrm{RbF}$.

\section{HAMILTONIAN AND ENERGY LEVELS}

In the presence of very weak external electric and magnetic fields, the Hamiltonian for a polar diatomic molecule in a ${ }^{1} \Sigma$ electronic ground state, which has one nucleus with spin $\frac{1}{2}$ and one nucleus with spin $>\frac{1}{2}$ is $^{10}$

$$
\begin{aligned}
& \tilde{H}=F_{v, J^{\mathrm{op}}}-e Q_{1} q_{1} \frac{3\left(\mathbf{I}_{1} \cdot \mathbf{J}\right)^{2}+\frac{3}{2}\left(\mathbf{I}_{1} \cdot \mathbf{J}\right)-\mathbf{I}_{1}^{2} \mathbf{J}^{2}}{2 I_{1}\left(2 I_{1}-\mathbf{1}\right)(2 J-1)(2 J+3)}+c_{1} \mathbf{I}_{1} \cdot \mathbf{J}+c_{2} \mathbf{I}_{2} \cdot \mathbf{J} \\
& +c_{3} \frac{3\left(\mathbf{I}_{1} \cdot \mathbf{J}\right)\left(\mathbf{I}_{2} \cdot \mathbf{J}\right)+3\left(\mathbf{I}_{2} \cdot \mathbf{J}\right)\left(\mathbf{I}_{1} \cdot \mathbf{J}\right)-2\left(\mathbf{I}_{1} \cdot \mathbf{I}_{2}\right) J(J+1)}{(2 J-1)(2 J+3)}+c_{4} \mathbf{I}_{1} \cdot \mathbf{I}_{2}-\mathbf{u} \cdot \mathbf{E} \\
& -\mu_{N} g_{J}(\mathbf{J} \cdot \mathbf{H})-\mu_{N} g_{1}\left(\mathbf{I}_{1} \cdot \mathbf{H}\right)-\mu_{N} g_{2}\left(\mathbf{I}_{2} \cdot \mathbf{H}\right),
\end{aligned}
$$

where the subscript 1 refers to the nucleus with spin $>\frac{1}{2}$ and the subscript 2 refers to the remaining nucleus. For CsF, 1 and 2 are to be identified with the cesium and fluorine nuclei, respectively; the cesium nucleus has spin $I_{1}=\frac{7}{2}$. Briefly, the terms appearing in the Hamiltonian have the following significance: the first term is the operator for vibration and rotation; the second term describes the electric-quadrupole interaction for the cesium nucleus; the third and fourth terms represent the spin-rotation interactions for each nucleus; and the fifth and sixth terms are the tensor and scalar parts, respectively, of the nuclear spin-spin interaction ${ }^{11}$ which is due to both the direct nuclear dipole-dipole interaction and the electron-coupled interactions between the two nuclear spins. The remaining terms arise when the interactions between external fields and the electric and magnetic moments of the molecule are taken into consideration.

For ${ }^{1} \Sigma$ molecules with quadrupole hfs from only one nucleus, the representation $\left(J, I_{1}, F_{1}, I_{2}, F, M_{F}\right)$, frequently abbreviated by $\left(F_{1}, F\right)$, is suitable for the description of MBER spectra taken in very weak external fields. In the previous work on $\mathrm{CsF}$, the magnetic interactions of the fluorine nucleus have been adequately described by matrix elements of the $c_{2}\left(\mathbf{I}_{2} \cdot \mathbf{J}\right)$ interaction and the direct nuclear spin-spin interaction (see Sec. VI) which are diagonal in all quantum numbers. For this experiment, however, it is necessary to include both the tensor and scalar parts of the electron- coupled nuclear spin-spin interaction; moreover the magnetic interaction of the fluorine nucleus causes small but significant deviations from pure $\left(F_{1}, F\right)$ coupling, so matrix elements of the $c_{2}\left(\mathbf{I}_{2} \cdot \mathbf{J}\right)$ and spinspin interactions which are not diagonal in $F_{1}$ must be taken into account. The additional deviations from pure coupling which can arise from quadrupole matrix elements not diagonal in $J$ are well known ${ }^{12}$; the quadruple coupling energies in $\mathrm{CsF}$ are very much smaller than the separation between rotational states, however, so this effect has a magnitude on the order of $1 \mathrm{~Hz}$ and is completely negligible for our work.

Using the expressions for the hfs energies given in the appendix, the zero-field frequencies of the hfs transitions within the $J=1$ rotational level of a molecule in the $v$ th vibrational state can be expressed as a function of the coupling constants $c_{0}\left(\equiv e Q_{1} q_{1}\right), c_{1}, c_{2}, c_{3}$, and $c_{4}$ which appear in the molecular Hamiltonian:

$$
f_{k}(v)=\sum_{i=0}^{\ell} G_{k i} c_{i}(v)+f_{k}^{\prime}(v) .
$$

In this expression, $f_{k}$ is the frequency of the $k$ th transition (see Fig. 1), and the $G_{k i}$ are known functions of the angular momentum quantum numbers which characterize the initial and final states. The off-diagonal parts of the magnetic interaction of the fluorine nucleus contribute an amount $f_{k}{ }^{\prime}$ to the $k$ th zero-field transition frequency. The $f_{k}^{\prime}$ are nonlinear functions of the $c_{i}^{\prime}$ 's and amount to about $1 \%$ of the $f_{k}$, so their contribu-

${ }^{4}$ G. Bemski, W. A. Nierenberg, and H. B. Silsbee, Phys. Rev. 98, 470 (1955)

F. Mehran, R. A. Brooks, and N. F. Ramsey, Phys. Rev. 141, 93 (1966).

- A. Honig, M. Mandel, M. L. Stitch, and C. H. Townes, Phys. Rev. 96, 629 (1954).

7 G. Gräff and Ö. Runólfsson, Z. Physik 187, 140 (1965).

8 T. C. English, Ph.D. thesis, University of Michigan, 1966 (University Microfilms, Inc., Ann. Arbor, 1966).

${ }^{9}$ J. C. Zorn, T. C. English, J. T. Dickinson, and D. A. Stephenson, J. Chem. Phys. 45, 3731 (1966),

${ }_{10} \mathrm{P}$. Kusch and V. W. Hughes, Encyclopedia of Physics (Springer-Verlag, Berlin, 1959), Vol. 37/1, p. 124. The Hamiltonian given in this article does not include the nuclear spin-spin interaction in its most general form; this interaction is discussed by N. F. Ramsey, Phys. Rev. 91, 303 (1953) and C. Schlier, Fortschr. Physik 9, 455 (1961).

${ }_{11}$ The relation between $c_{3}$ and $c_{4}$, and the quantities $\dot{d}_{T}$ and $d_{S}$ of Gräff and Runólfsson (Ref. 7 ) is: $c_{3}=d_{T}, c_{4}=d_{S}$.

${ }_{12}$ C. H. Townes and A. L. Schawlow, Microwave Spectroscopy (McGraw-Hill Book Co., New York, 1955), pp. 15-16, 155-164, $517-521$. 


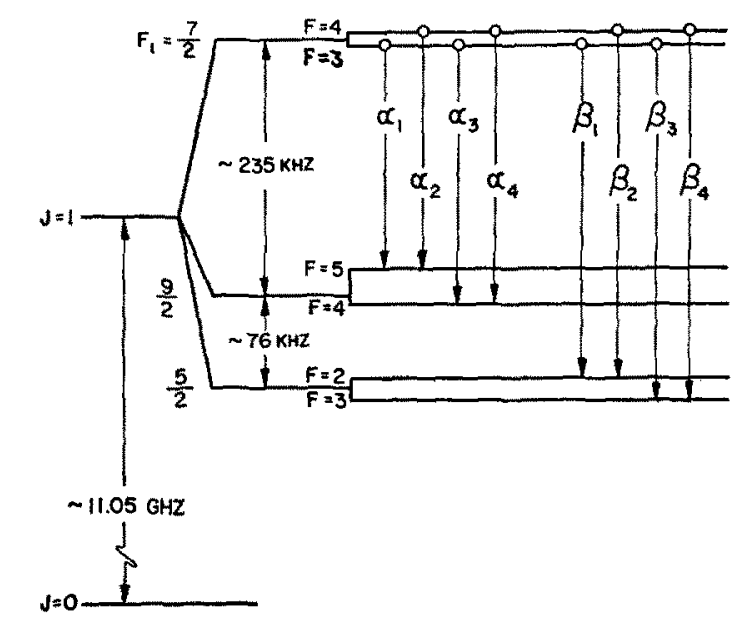

FIG. 1. Zero-field hyperfine structure of the $J=1$ rotational level of ${ }^{\mathrm{C}} \mathrm{Cs}^{10} \mathrm{~F}$.

tions are easily seen with the resolution of the present apparatus.

Single quantum transitions of the form $\left(F_{1}, F\right) \rightarrow$ $\left(F_{1}^{\prime}, F^{\prime}\right)$ are forbidden in zero electric field, ${ }^{13}$ so it is necessary to relate the observed frequencies $f_{k}^{\text {obs }}$ of the lines as measured in very small (but finite) fields to the corresponding zero-field frequencies $f_{k}$ as given by Eq. (2) in order to calculate the hfs constants from the observed spectrum.

\section{SPECTRA}

\section{A. Observations}

The work of Trischka ${ }^{2}$ shows that the very-weakfield, $J=1$ spectrum of $\mathrm{CsF}$ consists of line groups near 230 and $310 \mathrm{kHz}$, and that the ordering of the $F_{1}$ levels in zero field is as shown in Fig. 1. The splitting of each $F_{1}$ level by the magnetic interaction of the fluorine nucleus is small compared to the spacing between the $F_{1}$ levels, so the entire $F_{1}=\frac{7}{2}$ level lies well above the $F_{1}=\frac{5}{2}$ and $F_{1}=\frac{9}{2}$ levels. The observability criterion ${ }^{13}$ then shows that only the transitions out of the $F_{1}=\frac{7}{2}$ level should give signal changes at the detector, thus eight spectral lines, indicated in Fig. 1, are expected for each vibrational state. The use of sequential oscillating fields to increase the number of observed lines is discussed in the following paper. ${ }^{14}$

Eight lines were observed for the $v=0$ state; six were clearly resolved, one was partially resolved, and one appeared only as a slight bulge on the side of one of the $v=1$ lines. The center frequencies of these lines and uncertainties in determining them are given in Table $\mathrm{I}$. The frequency range $150-330 \mathrm{kHz}$ was searched with

\footnotetext{
${ }_{13}$ V. Hughes and L. Grabner, Phys. Rev. 79, 829 (1950). Diabatic transitions in the regions between the state selectors and the $C$ field may permit the observation of if-induced transitions which do not satisfy the usual observability criterion; these were looked for but not found in the present experiment. ${ }_{14} J$. C. Zorn, D. Stephenson, J. T. Dickinson, and T. C. English, J. Chem. Phys. 47, 3904 (1967), following paper.
}

particular emphasis being given to the expected locations of the $J=2$ spectrum, but no lines other than those attributable to $J=1$ were seen.

Not all the expected lines were recorded for vibrational states $v=1$ and $\nu=2$ because of the lower number of beam molecules in these higher vibrational states and because of partial overlapping of these spectra with the $v=0$ spectrum as well as with each other. The identification of the spectral lines seen in the experiment was made difficult by this overlapping, but the line assignments have been confirmed by a subsequent triple resonance study ${ }^{14}$ on $\mathrm{CsF}$.

Five relatively clean lines were seen for $v=1$, and four for $v=2$. The displacement of each line from its counterpart in the preceding vibrational state $(v$ less by 1) was measured by separately plotting the corresponding lines and then overlaying the two curves and aligning them so that they were as nearly coincident as possible. This method of determining frequency differences has the advantage of matching the curves at a large number of points and thus a good determination of the vibrational state dependence of the hfs constants can be made. Table II lists frequency differences obtained in this way.

\section{B. Effect of External Fields}

In this experiment it is very important that the external fields be kept small enough that the observed spectra can be simply and accurately interpreted using the values of the zero-field frequencies as calculated from the molecular Hamiltonian. Unfortunately, the transition probability for single quantum transitions becomes vanishingly small in the limit of zero dc electric field, so the normal operating values of the $\mathrm{dc}$ and rf electric fields of $1.5 \mathrm{~V} / \mathrm{cm}$ and $0.4 \mathrm{~V} / \mathrm{cm} \mathrm{pp}$, respectively, represent a compromise between adequate signal-to-noise ratio and fulfillment of the very-weakfield criterion. The magnetic field over the entire $C$ region is reduced to less than 0.05 Oe by cancelling the earth's magnetic field with a set of coils.

TaBLE I. Hfs transition frequencies ${ }^{\mathrm{a}} \mathrm{b}$ in the $J=1, v=0$ state of ${ }^{133} \mathrm{Cs}^{18} \mathrm{~F}$.

\begin{tabular}{lcccc}
\hline \hline & $\begin{array}{c}\text { Transition } \\
\left(F_{1}, F\right) \rightarrow\left(F_{1}^{\prime}, F^{\prime}\right)\end{array}$ & $\begin{array}{c}\text { Observed } \\
\text { frequency }\end{array}$ & $\begin{array}{c}\text { Calculated } \\
\text { frequency }\end{array}$ & $\begin{array}{c}\text { Weighting } \\
\text { factor, } w_{k}\end{array}$ \\
\hline$\alpha_{1}$ & $(7 / 2,3)-(9 / 2,5)$ & $224.60(25)$ & 224.71 & 0.5 \\
$\alpha_{2}$ & $(7 / 2,4)-(9 / 2,5)$ & $228.05(15)$ & 227.99 & 1.0 \\
$\alpha_{3}$ & $(7 / 2,3)-(9 / 2,4)$ & $245.80(25)$ & 245.78 & 0.8 \\
$\alpha_{4}$ & $(7 / 2,4)-(9 / 2,4)$ & $249.05(20)$ & 249.06 & 0.9 \\
& & & & \\
$\beta_{1}$ & $(7 / 2,3)-(5 / 2,2)$ & $304.90(20)$ & 304.86 & 0.9 \\
$\beta_{2}$ & $(7 / 2,4)-(5 / 2,2)$ & $308.10(15)$ & 308.14 & 0.9 \\
$\beta_{3}$ & $(7 / 2,3)-(5 / 2,3)$ & $314.40(30)$ & 314.33 & 0.1 \\
$\beta_{4}$ & $(7 / 2,4)-(5 / 2,3)$ & $317.60(15)$ & 317.61 & 1.0 \\
\hline
\end{tabular}

a All frequencies in kilohertz.

b Numbers in parentheses are uncertainties in units of the last digit. 
From measurements on several lines, we have determined that the Zeeman shifts of the apparent line centers are less than $200 \mathrm{~Hz}$ when the field is increased by $0.06 \mathrm{Oe}$ above the normal operating value; moreover the general shape and width of the lines do not appear to depend noticeably on magnetic field when it has a magnitude of less than 0.1 Oe.

The dependence of line shape and position on $\mathrm{rf}$ electric field strength becomes noticeable above $E_{\mathrm{rf}} \simeq 1.0$ $\mathrm{V} / \mathrm{cm}$ pp when a $1.5-\mathrm{V} / \mathrm{cm}$ dc field is also present; reduction of the rf level below the normal $E_{\text {rf }} \simeq 0.4$ $\mathrm{V} / \mathrm{cm} \mathrm{pp}$ reduces the amplitude of the lines but does not affect their shape in a noticeable way.

Static electric fields in excess of $3.0 \mathrm{~V} / \mathrm{cm}$ distort and shift the spectral lines in a very noticeable way, so the normal operating field is kept at $1.5 \mathrm{~V} / \mathrm{cm}$. Reduction of the field to $1.0 \mathrm{~V} / \mathrm{cm}$ shifts a line center by less than $150 \mathrm{~Hz}$,typically, but the width of the line may still decrease slightly as the value of the electric field is reduced. For example, a reduction of the field from 15 to $1.0 \mathrm{~V} / \mathrm{cm}$ reduces the width of the $317.6-\mathrm{kHz}$ line from 1.0 to $0.7 \mathrm{kHz}$, as shown in Fig. 2. Calculations show that a dc field of $1.5 \mathrm{~V} / \mathrm{cm}$ will yield Stark components of the $317.6-\mathrm{kHz}$ lines which are removed by as far as +540 and $-400 \mathrm{~Hz}$ from the zero-field position, while a $1.0-\mathrm{V} / \mathrm{cm}$ field gives extreme displacements of +240 and $-180 \mathrm{~Hz}$. Thus the calculated separation between furthest displaced Stark components changes from 940 to $420 \mathrm{~Hz}$ as the external field drops from 1.5 to $1.0 \mathrm{~V} / \mathrm{cm}$; this is to be compared with the observed $300-\mathrm{Hz}$ reduction in width of the entire 317.6-kHz spectral line.

A reliable analysis of the lines observed in very-weakfield, MBER spectroscopy is difficult because the effects of diabatic transitions on the relative populations of the levels are not known, and because the transition probablities are not small enough to permit the use of perturbation theory for this many-level problem.

TABLE II. Displacements,b of $J=1 \mathrm{hfs}$ transition frequencies with vibrational state for ${ }^{138} \mathrm{Cs}^{19} \mathrm{~F}$.

\begin{tabular}{lccc}
\hline & $\begin{array}{c}\text { Transition } \\
\left(F_{1}, F\right) \rightarrow\left(F_{2}{ }^{\prime}, F^{\prime}\right)\end{array}$ & $\begin{array}{c}v=0 \rightarrow 1 \\
\text { Displacement }\end{array}$ & $\begin{array}{c}v=1 \rightarrow 2 \\
\text { Displacement }\end{array}$ \\
\hline$\alpha_{1}$ & $(7 / 2,3)-(9 / 2,5)$ & $2.50(5)$ & $\ldots$ \\
$\alpha_{2}$ & $(7 / 2,4)-(9 / 2,5)$ & $2.60(20)$ & $2.50(5)$ \\
$\alpha_{3}$ & $(7 / 2,3)-(9 / 2,4)$ & $2.80(10)$ & $2.80(10)$ \\
$\alpha_{4}$ & $(7 / 2,4)-(9 / 2,4)$ & $\cdots$ & $\cdots$ \\
$\beta_{1}$ & $(7 / 2,3)-(5 / 2,2)$ & $3.55(5)$ & $3.45(10)$ \\
$\beta_{2}$ & $(7 / 2,4)-(5 / 2,2)$ & $\cdots$ & $\cdots$ \\
$\beta_{3}$ & $(7 / 2,3)-(5 / 2,3)$ & $\cdots$ & $\cdots$ \\
$\beta_{4}$ & $(7 / 2,4)-(5 / 2,3)$ & $3.60(5)$ & $3.60(5)$ \\
\hline
\end{tabular}

all frequencies in kilohertz. All lines observed are shifted to lower fre quencies as $v$ is increased, i.e., each displacement listed above is the amount by which the frequency of the given transition in the state $(\eta+1)$ is less than that of the same transition in the state $v$.

$b$ Numbers in parentheses are uncertainties in units of the last digit.

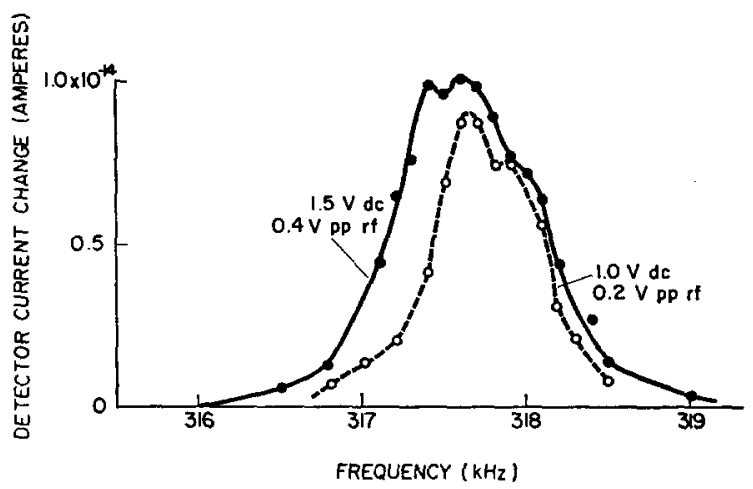

FIG. 2. Effect of reducing the dc and rf voltages on the lineshape of the $\beta_{4}\left[\left(\frac{7}{2}, 4\right)-\left(\frac{5}{2}, 3\right)\right]$ transition.

\section{ANALYSIS OF DATA}

The coupling constants of the $v=0$ state are obtained by using the eight observed transition frequencies $f_{k}^{\text {obs }}$ in a least-squares analysis to choose that set of hfs constants $c_{i}$ which minimizes the quantity,

$$
\sum_{k=1}^{s}\left(f_{k}^{\text {obs }}-f_{k}\right)^{2} w_{k},
$$

where $w_{k}$ is a subjective weighting factor which gives our estimate of the quality of $f_{k}{ }^{\text {obs }}$, and the $f_{k}$ are obtained from Eq. (2).

Since the $f_{k}{ }^{\prime}$ in Eq. (2) are nonlinear functions of the $c_{i}$, it is convenient to carry out the least-squares analysis using a method of successive approximations. Initially we set $f_{k}{ }^{\prime}=0$ and minimize expression (3), obtaining the $c_{i}$ in the first approximation. The weighted sum (3) is then minimized again, this time using values of the $f_{k}{ }^{\prime}$ calculated from the $c_{i}$ in the previous approximation. This process is repeated until there is no significant change in the $c_{i}$ from one approximation to the next; for our calculations only two iterations are required. Table I shows a comparison between the $v=0$ values of $f_{k}$ obs and $f_{k}$, and the agreement is excellent.

For $v=1$ and $v=2$, the hfs constants are not overdetermined because of the smaller number of lines observed. The $c_{i}$ for these states can be calculated by using the $f_{k}{ }^{\prime}$ found from the $v=0$ calculations together with the $f_{k}^{\text {obs }}(v)$ to write, in analogy with Eq. (2):

$$
f_{k}^{\mathrm{obs}}(v)-f_{k}{ }^{\prime}=\sum_{i=0}^{4} G_{k i} c_{i}(v) .
$$

The five lines observed in the $v=1$ state used in Eq. (4) form a system of simultaneous linear equations whose solution yields values for the $v=1 \mathrm{hfs}$ constants. Only four lines are seen for the $v=2$ state, so we assume $c_{4}(v=2)=c_{4}(v=0)$ [based on the observation that our values for $c_{4}(v=0)$ and $c_{4}(v=1)$ differ by much less than their individual uncertainties] and solve Eq. (4) for the remaining coupling constants; fortuitously, our values for these $v=2 \mathrm{hfs}$ constants are very insensitive to the assumed value of $c_{\mathbf{4}}$. Iteration of the system of 
TABLE III. Hfs constants ${ }^{\mathrm{a}, \mathrm{b}}$ for the $J=1$ state of ${ }^{133} \mathrm{Cs}^{19} \mathrm{~F}$.

\begin{tabular}{|c|c|c|c|c|c|c|}
\hline & & $e Q_{1} q_{1}$ & $c_{1}$ & $c_{2}$ & $c_{3}$ & $c_{4}$ \\
\hline Present experiment MBER, very weak field & $\begin{array}{l}v=0 \\
y=1 \\
y=2\end{array}$ & $\begin{array}{l}1.2370(13) \\
1.2230(14) \\
1.2090(14)\end{array}$ & $\begin{array}{l}0.70(7) \\
0.68(8) \\
0.66(8)\end{array}$ & $\begin{array}{l}15.1(6) \\
15.0(6) \\
14.7(6)\end{array}$ & $\begin{array}{l}0.92(12) \\
0.90(13) \\
0.89(13)\end{array}$ & $\begin{array}{l}0.61(10) \\
0.62(24)\end{array}$ \\
\hline MBER, strong magnetic and electric fields ${ }^{e}$ & $\begin{array}{l}y=0 \\
v=1\end{array}$ & $\begin{array}{l}1.2383(6) \\
1.224(5)\end{array}$ & $0.64(2)$ & $14.94(6)$ & $0.94(4)$ & $\left|c_{4}\right|<5$ \\
\hline MBER, strong and weak electric field ${ }^{d}$ & $\nu=0$ & $1.240(8)$ & $0(1)$ & $16(2)$ & & \\
\hline Molecular beam magnetic resonance $e^{e, t}$ & & & & $12.0(6)$ & & \\
\hline Molecular beam magnetic resonance $e^{t, \mathbf{s}}$ & & & & $13.5(25)$ & & \\
\hline
\end{tabular}

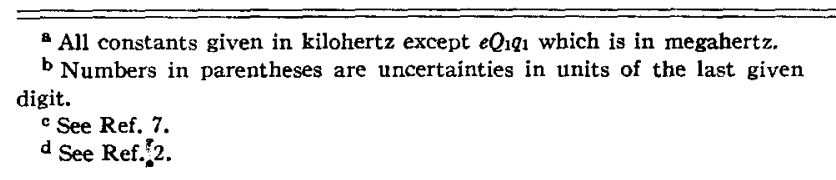

equations, Eq. (4), using $f_{k}{ }^{\prime}$ formed from $c_{i}(v>0)$ does not produce any significant changes in the values of the $c_{i}$ obtained for $v>0$, so the approximation $f_{k}^{\prime}(v)=f_{k}{ }^{\prime}(0)$ is satisfactory for analysis of this experiment.

The uncertainty in each of the hfs coupling constants is estimated by inverting the system of equations, Eq. (4), in order to show how the values of the hfs constants depend on the observed frequencies:

$$
c_{i}=\sum_{k=1}^{5} G_{i k}{ }^{-1}\left(f_{k}^{\text {obs }}-f_{k}{ }^{\prime}\right),
$$

where the five $f_{k}^{\text {obs }}$ are chosen from the total number available for the vibrational state of interest. The total uncertainty in the $i$ th hfs constant, $\Delta c_{i}$, is taken to be

$$
\Delta c_{i}=\left[\sum_{k=1}^{5}\left(G_{i k}^{-1} \delta f_{k}\right)^{2}\right]^{1 / 2}+\epsilon \sum_{k=1}^{5}\left|G_{i k}^{-1}\right|
$$

where $\delta f_{k}$ is the statistical uncertainty in $f_{k}^{\text {obs }}$ (from scatter in the values from many observations of the same line) and $\epsilon$ is our estimate for systematic error which may arise from the Stark and Zeeman effects. The quantity $\epsilon$ is judged to be about $200 \mathrm{~Hz}$ for all lines and we believe that it is the main factor limiting the precision to which the hfs constants can be determined from our MBER measurements.

For the ground vibrational state, for which eight spectral lines are measured, we choose for each $c_{i}$ that set of five $f_{k}$ obs which gives the smallest value of $\Delta c_{i}$. There are only five lines observed for $v=1$, so we use them all. For $v=2$, we assume a value of $c_{4}$ and estimate the remaining $\Delta c_{i}$ as for $v=1$.

\section{RESULTS}

The values of the hfs constants as determined from the spectra for the first three vibrational states of $\mathrm{CsF}$ are given in Table III along with the values found in previous experiments. The values of the spin-rotation constant $c_{2}$ from all the electric resonance experiments

\author{
- See Ref. 4. \\ $f$ Magnetic resonance values of $c_{2}$ are averages over vibrational and rota- \\ tional states. \\ See Ref. 5.
}

and from the magnetic resonance molecular beam experiment of Mehran, Brooks, and Ramsey ${ }^{5}$ are consistent with one another; the disagreement between these values and the value of $c_{2}$ obtained from the magnetic resonance molecular beam experiment of Bemski, Nierenberg, and Silsbee ${ }^{4}$ is not understood, but might be explained if $c_{2}$ were to possess even a small dependence on the rotational quantum number $J$. Such an effect has been observed ${ }^{15}$ in diatomic fluorine, and it is known that molecules in a wide range of $J$ states contribute to the spectra of alkali halides as measured with a magnetic resonance beam spectrometer.

For the hfs constants other than $c_{2}$ the only values available for comparison are those from the two other electric resonance experiments. Both the values from our experiment and the strong-field experiment of Gräff and Runólfsson are in agreement with the earlier, less accurate work of Trischka, but the most significant comparison is between the former two experiments. In this regard, our values of $c_{1}, c_{2}$, and $c_{3}$ for the $v=0$ state are in good agreement with the values of Gräff and Runólfsson; comparison of the values of these same constants for the $v=1,2$ states is not possible since the present experiment is the first to measure them. The values of $c_{4}$ for $v=0,1$ are also given here for the first time, the $v=0$ value confirming the observation of Gräff and Runólfsson that its magnitude is less than $5 \mathrm{kHz}$. The values for the quadrupole coupling constants obtained from the two experiments overlap within the combined, quoted uncertainties, but the disagreement between the quoted central values for $e Q_{1} q_{1}$ may prove to be of significance if very detailed studies of quadrupole hfs are undertaken in future work. For $v=1$ the values of $e Q_{1} q_{1}$ are in good agreement but this is less significant in view of the increased experimental uncertainties relative to $v=0$.

${ }_{15}^{15}$ I. Ozier, Ph.D. thesis, Harvard University, 1965 (unpublished); I. Ozier and N. F. Ramsey, Bull. Am. Phys. Soc. 11, 23 (1966). 
TABLE IV. Values of spin-spin interaction constants for the alkali fluorides, ${ }^{\mathrm{B}, \mathrm{b}}$

\begin{tabular}{|c|c|c|c|c|c|}
\hline Molecule & $c_{3}$ & $c_{4}$ & $\left(c_{3}\right)_{\mathrm{dir}}$ & $\left(c_{3}\right)_{\mathrm{eo}}$ & Reference \\
\hline${ }^{133} \mathrm{Cs}^{19} \mathrm{~F}$ & $\begin{array}{l}0.92(8) \\
0.94(4)\end{array}$ & $\begin{array}{l}0.61(9) \\
\cdots\end{array}$ & $\begin{array}{l}1.15 \\
1.15\end{array}$ & $\begin{array}{l}-0.23(8) \\
-0.21(4)\end{array}$ & $\begin{array}{l}c \\
d\end{array}$ \\
\hline${ }^{85} \mathrm{Rb}^{19} \mathrm{~F}$ & $\begin{array}{l}0.80(8) \\
0.93(5)\end{array}$ & $\begin{array}{l}0.15(8) \\
0.23(6)\end{array}$ & $\begin{array}{l}0.93 \\
0.93\end{array}$ & $\begin{array}{l}-0.8(8) \\
-0.00(5)\end{array}$ & $\begin{array}{l}\mathrm{e} \\
\mathrm{f}\end{array}$ \\
\hline${ }^{87} \mathrm{Rb}^{19} \mathrm{~F}$ & $3.16(18)$ & $0.66(10)$ & 3.15 & $0.01(18)$ & $\mathrm{f}$ \\
\hline${ }^{39} \mathrm{~K}^{19} \mathrm{~F}$ & $0.29(12)$ & 0.3 or -0.6 & 0.51 & $-0.22(12)$ & $\mathrm{g}$ \\
\hline${ }^{23} \mathrm{Na}^{19} \mathrm{~F}$ & $\begin{array}{l}3.85(25) \\
3.7(2)\end{array}$ & $\begin{aligned} & 0.0(4,-1) \\
- & 0.2(2)\end{aligned}$ & $\begin{array}{l}4.16 \\
4.16\end{array}$ & $\begin{array}{l}-0.31(25) \\
-0.46(20)\end{array}$ & $\mathrm{h}$ \\
\hline${ }^{7} \mathrm{Li}^{19} \mathrm{~F}$ & $\begin{array}{c}11.390(15) \\
0.42(6)\end{array}$ & $\begin{array}{l}0.21(4) \\
0.06(20)\end{array}$ & $\begin{array}{r}11.33 \\
0.51\end{array}$ & $\begin{array}{l}0.06 \\
-0.09(6)\end{array}$ & $\begin{array}{l}\mathbf{j} \\
\mathbf{k}\end{array}$ \\
\hline
\end{tabular}

a All constants are in kilohertz and are for $J=1 y=0$ molecules.

b Numbers in parentheses are uncertainties in units of the last digit.

c Present work.

d See Ref. 7.

- See Ref. 9.

${ }^{f}$ P. A. Bonczyk and V. W, Hughes, Phys. Rev, 161, 15 (1967).

E G. Gräff and O. Runblfsson, Z. Physik 176, 90 (1963), particularly p. 108 .

\section{DISCUSSION}

From the experimental value for the constant $c_{3}$, it is possible to determine the tensor part of the electroncoupled nuclear spin-spin interaction, $\left(c_{3}\right)_{\mathrm{ec}}$. To do this, we use the relation $c_{3}=\left(c_{3}\right)_{\mathrm{dir}}+\left(c_{3}\right)_{\mathrm{ec}}$, where $\left(c_{3}\right)_{\mathrm{dir}}$ is the direct nuclear spin-spin interaction given by $\left(c_{3}\right)_{\mathrm{dir}}=g_{1} g_{2} \mu_{N}{ }^{2}\left\langle v, J\left|r^{-3}\right| v, J\right\rangle$. In this latter expression, $g_{1}$ and $g_{2}$ are the nuclear $g$ factors, $\mu_{N}$ is the nuclear magneton, $r$ is the internuclear distance, and $\left\langle v, J\left|r^{-3}\right| v, J\right\rangle$ is the expectation value of $r^{-3}$ in vibrational state $v$ and rotational state $J$. For a Morse potential, this latter quantity may be evaluated ${ }^{3,16}$ for $v=0$ and $J=1$ to give $0.996 r_{e}^{-3}$ so that $\left(c_{3}\right)_{\mathrm{dir}}=1.15$ $\mathrm{kHz}$. This yields the desired quantity $\left(c_{3}\right)_{\mathrm{ec}}=-0.23(12)$ $\mathrm{kHz}$ which is to be compared with the scalar part of the electron-coupled interaction, $c_{4}=+0.61(10) \mathrm{kHz}$. For purposes of comparison, the values of $c_{3},\left(c_{3}\right)_{\mathrm{dir}}$, $\left(c_{3}\right)_{e e}$, and $c_{4}$ for the other alkali fluorides are sum. marized in Table IV.

The present experiment also provides information on the vibrational state dependence of the hfs constants. Since the Stark and Zeeman effects from very weak fields for any given $\left(F_{1}, F\right) \rightarrow\left(F_{1}^{\prime}, F^{\prime}\right)$ transition are the same (to within our experimental accuracy) for molecules in different vibrational states, the determination of the relative change in the coupling constants from one vibrational state to the next is not dependent on minute details of the line shape and can be determined by comparing the frequencies of corresponding lines in the spectra of the vibrational states in question. For example, the variation in $e Q_{1} q_{1}$ with $v$ can be calculated almost directly from the frequency differences given in Table II; the result is that

$$
\begin{aligned}
& e Q_{1} q_{1}(v=1)-e Q_{1} q_{1}(v=0)=-0.01415(24) \mathrm{MHz}, \\
& e Q_{1} q_{1}(v=2)-e Q_{1} q_{1}(v=1)=-0.01396(16) \mathrm{MHz} .
\end{aligned}
$$

${ }^{16}$ S. E. Veazey and W. Gordy, Phys. Rev. 138, A1303 (1965). b R. Van Wachem and A. Dymanus, J. Chem. Phys. 46, 3749 (1967).

i C. D. Hollowell, A. J. Hebert, and K. Street, Jr., J. Chem. Phys. 4I, 3540 (1964).

j G. Gräff and G. Werth, Z. Physik 183, 223 (1965).

k L. Wharton, L. P. Gold, and W. Klemperer, Phys, Rev, 133, B270 (1964).

These numbers represent changes in $e Q_{1} q_{1}$ [relative to $\left.e Q_{1} q_{1}(v=0)\right]$ of $-1.14(2) \%$ and $-1.13(1) \%$, respectively.

These changes in $e Q_{1} q_{1}$ may be compared with those predicted from two models in which the electric field gradient $q_{1}$ at the site of the alkali nucleus varies with the internuclear separation as $r^{-n}$ (where $n$ is a positive integer greater than 1) and where it is assumed that the variation in $e Q_{1} q_{1}$ with vibrational state is due entirely to the variation in $q_{1}$.

The first of these is the Sternheimer antishielding theory ${ }^{17}$ in which the gradient $q$ at the nucleus of a free ion (which has no partially filled electron shells) due to a point charge $-e$ a distance $r$ away is given by

$$
q=\left(-2 e / r^{3}\right)[1-\gamma(r)]
$$

where $\gamma(\mathbf{r})$ is the antishielding factor for the ion. To obtain an expression for $q$ at the alkali nucleus in an alkalifluoride molecule, in which the bonding is largely ionic, it is a good approximation to consider the fluorine negative ion to be equivalent to a point charge $-e$ located at the position of the fluorine nucleus. The quantity $r$ is then the internuclear distance. In the case of predominantly ionic bonding it is also usually a good approximation to take $\gamma(r)=\gamma(\infty)$ so that $q \propto r^{-3}$. For the $\mathrm{Cs}^{+}$ion, $\gamma(\infty)$ has been calculated ${ }^{17}$ to be -102.5 .

In what follows, it is convenient to define the quantity $\Delta$ to be the percent increase in $q$ (relative to $q$ for $v=0$ ) per vibrational state:

$$
\Delta \equiv\left[\left(\langle q\rangle_{v+1}-\langle q\rangle_{v}\right) /\langle q\rangle_{v=0}\right] 100,
$$

where $\langle q\rangle_{v}$ is the expectation value of $q$ in the $v$ th vibrational state. $\Delta$ for the above model may be eval-

17 R. M. Sternheimer, Phys. Rev. 80, 102 (1950); 84, 244 $(1951) ; 146,140(1966)$. R. M. Sternheimer and H. M. Foley, Phys. Rev. 102, 731 (1956). Additional references on the subject may be found in Ref. 18. We are indebted to Dr. Sternheimer for his helpful comments on our work. 
uated by assuming an internuclear potential of the Dunham form

$$
U(\boldsymbol{r})=a_{0} \xi^{2}\left[1+a_{1} \xi+\mathcal{O}\left(\xi^{2}\right)\right],
$$

where $\xi \equiv\left(r-r_{e}\right) / r_{e}$ from which the expectation value $^{3,18}$

$$
\left\langle r^{-n}\right\rangle_{v}=r_{e}^{-n}\left[1+n\left(B_{e} / \omega_{e}\right)\left(n+1+3 a_{1}\right)\left(v+\frac{1}{2}\right)\right]
$$

can be derived using perturbation theory. For the Sternheimer theory, $n=3$ so that the result is

$$
(\Delta)_{\text {sternheimer }}=\left(3 B_{e} / \omega_{e}\right)\left(4+3 a_{1}\right) \text {. }
$$

Using the values of $B_{e}, \omega_{e}$, and $a_{1}$ for $\mathrm{CsF}$ as determined by Veazey and Gordy ${ }^{16}$ this amounts to $-0.80 \%$ and has the same sign as the experimental variation in $e Q_{1} q_{1}$, but is $30 \%$ smaller in magnitude. This theoretical approach has been extended by de Wijn ${ }^{18}$ to include effects of mutual polarization of the two ion cores, but the refinements change the value of $\Delta$ to $-0.76 \%$ which slightly increases the discrepancy between theory and experiment. ${ }^{19}$ If the variation of $\gamma(r)$ with $r$ is taken into account [instead of assuming $\gamma(\boldsymbol{r})=\gamma(\infty)]$ by using the value of $\gamma(\boldsymbol{r})$ calculated for $\mathrm{Cs}^{+}$by Foley, Sternheimer, and Tycko, ${ }^{20}$ the value of $(\Delta)_{\text {Stemheimer }}$ changes from $-0.80 \%$ to $-0.71 \%$.

Another model has been proposed by Makhanek.21 His work, which does not consider antishielding, is based on the conjecture that the contribution to $q$ at the alkali nucleus is due solely to a distortion of the electron shell of the alkali ion brought about by the adjacent fluorine ion. Such a conjecture leads to an $r^{-2}$ dependence of $q$ on the internuclear distance so that

$$
(\Delta)_{\text {Makhanek }}=\left(6 B_{e} / \omega_{e}\right)\left(1+a_{1}\right),
$$

which is evaluated to be $-0.64 \%$ for CsF. This is about a factor of 2 smaller in magnitude than the experimental result of $\Delta=-1.13 \%$.

In the two models considered, the vibrational state variation comes from the dependence of $q$ on inverse powers of the internuclear distance, both models predicting a decrease in the magnitude of $q$ with increase in $v$. In the approximation that the molecule is a simple harmonic oscillator ( $\mathrm{SHO}$ ), the probability density of the oscillator is symmetric about $r=r_{e}$; however, the average value of $r^{n}$ is greater than $r_{e}^{-n}$ because the smaller values of $r$ influence the average of $r^{-n}$ more heavily than do the larger values. As $v$ is increased, the

\footnotetext{
${ }^{18} \mathrm{H}$. W. de Wijn, J. Chem. Phys. 44, 810 (1966).

${ }^{19}$ For the other alkali fluorides $\left(\mathrm{Rb}^{85 \mathrm{~F}}, \mathrm{~K}^{39} \mathrm{~F}, \mathrm{NaF}, \mathrm{Li}^{7} \mathrm{~F}\right)$, these comments also apply in slightly modified form: $\Delta$ due to antishielding is of the correct sign, but smaller in magnitude than the experimental values by $15 \%$, typically (except $\mathrm{NaF}$ which gives exact agreement); the corrections due to mutual polarization are all $1 \%$ or less of $(\Delta)$ sternhoimer

${ }^{20}$ H. M. Foley, R. M. Sternheimer, and D. Tycko, Phys. Rev. 93, 734 (1954).

${ }_{21}$ A. G. Makhanek, Opt. Spectry. (USSR) 9, 214 (1960).
}

amplitude of oscillation is increased so that even smaller values of $r$ occur with significant probability. The result is that $\left\langle r^{-n}\right\rangle_{v}$ increases with increasing $v$. On this basis, the magnitude of $q$ would be expected to increase in the higher vibrational states. This is opposite to what is observed experimentally, and indicates that the observed variation in $e Q_{1} q_{1}$ depends very significantly on higher-order terms in the internuclear potential.

Consider now the addition of a cubic term to the potential of the oscillator, the term $a_{0} a_{1} \xi^{3}$ in Eq. (9). Since $a_{0}$ is positive and $a_{1}$ is negative for all the alkali fluorides, the effect of this term is to make the side of the potential curve with $r>r_{e}$ less steep. This results in two effects ${ }^{22}$ : (1) The oscillator is more likely to be found at $r>r_{e}$. (2) The classical turning point for $r>r_{e}$ is further from $r_{e}$ than is the other turning point. The larger values of $r$ are now favored on two accounts so that the average of $r^{-n}$ is smaller than for the SHO. Also, the above two effects become more pronounced as $v$ is increased so that there is a tendency for $\left\langle r^{-n}\right\rangle_{v}$ to decrease. Clearly, if the cubic term is sufficiently large, this decrease can overwhelm the increase in $\left\langle r^{-n}\right\rangle_{v}$ which occurs for the SHO to give a net decrease in $\left\langle r^{n}\right\rangle_{v}$ as $v$ is increased. This is just what happens in all the alkali fluorides, for which $a_{1} \simeq-3$. Mathematically, this is reflected in Eqs. (11) and (12) by the fact that $\Delta$ is negative only if $a_{1}<-\frac{4}{3}$ and -1 , respectively. Physically, then, the observed decrease of $\left|e Q_{1} q_{1}\right|$ with vibrational state is due to the asymmetry of the internuclear potential.

\section{ACKNOWLEDGMENTS}

We are grateful for helpful discussions with Professor P. R. Fontana, Professor P. A. Franken, Professor K. T. Hecht, Professor T. M. Sanders, and Professor G. Weinreich. We are indebted to J. T. Dickinson and D. A. Stephenson for their help in the final stages of the experiment and to Mrs. D. A. Stephenson for assistance with computer calculations.

\section{APPENDIX}

The zero-field energies for $J=1$ and arbitrary $v$ are obtained using degenerate perturbation theory in which the $F_{v, J}$ term in the Hamiltonian of Eq. (1) is taken to be the unperturbed Hamiltonian and all remaining terms (with $E=H=0$ ) are considered as a single perturbation $V$. The hfs energies to first order are obtained by the usual procedure of solving the secular determinant for the perturbation matrix corresponding to the degenerate unperturbed level of interest, which in the present case is the $J=1$ rotational level.

The evaluation of both diagonal and off diagonal matrix elements of $V$ is most easily accomplished using the techniques of Racah and others which may be found

\footnotetext{
${ }^{22}$ G. Herzberg, Spectra of Diatomic Molecules (D. Van Nostrand Co., Inc., Princeton, N.J., 1950), pp. 90-94.
} 
in the book by Edmonds. ${ }^{23}$ The result for matrix elements which are diagonal in $J$ is:

$$
\begin{aligned}
& \left\langle J, I_{1}, F_{1}, I_{2}, F, M_{F}|V| J, I_{1}, F_{1}{ }^{\prime}, I_{2}, F^{\prime}, M_{F}{ }^{\prime}\right\rangle \\
& =\left(-e Q_{1} q_{1}\left[\frac{(3 / 2) K_{1}\left(K_{1}+1\right)-2 I_{1}\left(I_{1}+1\right) J(J+1)}{4 I_{1}\left(2 I_{1}-1\right)(2 J-1)(2 J+3)}\right] \delta_{F_{1} F_{1}^{\prime}}+c_{1} \frac{1}{2} K_{1} \delta_{F_{1} F_{1}^{\prime}}\right. \\
& +C_{2}(-1)^{\left[J+I_{1}+2 F_{1^{\prime}}+I_{2}+F+1\right]}\left\{J(J+1)(2 J+1) N S_{2}\right\}^{1 / 2}\left\{\begin{array}{lll}
F & I_{2} & F_{1} \\
1 & F_{1}^{\prime} & I_{2}
\end{array}\right\}\left\{\begin{array}{lll}
J & F_{1} & I_{1} \\
F_{1}^{\prime} & J & 1
\end{array}\right\} \\
& +c_{3}(-1)^{\left[F_{1}^{\prime}+I_{2}+F+1\right]}\left[\frac{30 J(J+1)(2 J+1)}{(2 J+3)(2 J-1)} N S_{1} S_{2}\right]^{1 / 2}\left\{\begin{array}{lll}
F & I_{2} & F_{1} \\
1 & F_{1}^{\prime} & I_{2}
\end{array}\right\}\left\{\begin{array}{ccc}
J & J & 2 \\
I_{1} & I_{1} & 1 \\
F_{1} & F_{1}{ }^{\prime} & 1
\end{array}\right\}
\end{aligned}
$$

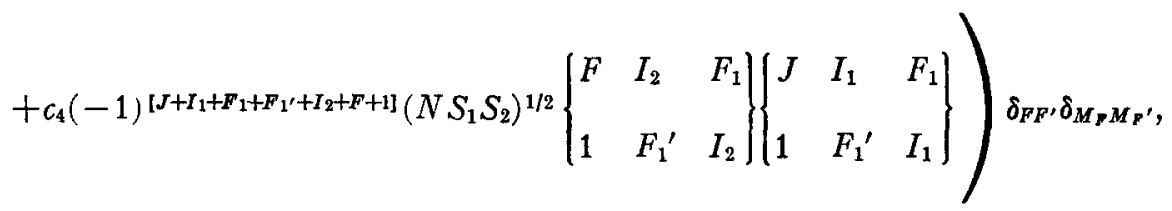

where

$$
\begin{aligned}
K_{1} & \equiv F_{1}\left(F_{1}+1\right)-I_{1}\left(I_{1}+1\right)-J(J+1), \\
N & \equiv\left(2 F_{1}+1\right)\left(2 F_{1}^{\prime}+1\right), \\
S_{1} & \equiv I_{1}\left(I_{1}+1\right)\left(2 I_{1}+1\right), \\
S_{2} & \equiv I_{2}\left(I_{2}+1\right)\left(2 I_{2}+1\right),
\end{aligned}
$$

and $J$ is restricted to integer values. Numerical evaluation of these matrix elements is most easily carried out using tables ${ }^{24}$ of the $3-j$ and $6-j$ symbols. The $9-j$ symbol can be evaluated in terms of $6-j$ symbols using the identities given in Edmonds.

The advantage of Eq. (A1) is that it provides a compact, general expression for the matrix elements of $V$, both diagonal and nondiagonal in the quantum numbers $F_{1}, F$, and $M_{F}$. In addition, the off-diagonal elements of $V$ contain contributions from the spin-spin interactions which are given here for the first time. Special cases of Eq. (A1) appear throughout the earlier literature: The diagonal matrix elements have been given previously ${ }^{13,25}$; moreover, the off-diagonal matrix elements of the $I_{2} \cdot J$ and $I_{1} \cdot I_{2}$ terms in $V$ may be found in the papers by Bardeen and Townes, ${ }^{26}$ and Zeiger and Bolef, ${ }^{25}$ respectively.

In our case $\left(I_{1}=\frac{7}{2}, I_{2}=\frac{1}{2}\right)$ the $J=1$ matrix is a

${ }^{23}$ A. R. Edmonds, Angular Momentum in Quantum Mechanics (Princeton University Press, Princeton, N.J., 1960), 2nd ed.

${ }^{21} \mathrm{M}$. Rotenberg, R. Bivins, N. Metropolis, and J. K. Wooten, Jr., The 3-j and 6-j Symbols (The Technology Press, Cambridge, Mass., 1959).

${ }_{25}$ H. J. Zeiger and D. I. Bolef, Phys. Rev. 85, 788 (1952). The second equation for the matrix element of $I_{a} \cdot I_{b}$ on $p .797$ contains a misprint; the factor $\left(F_{1}+I_{a}-J+1\right)^{1 / 2}$ which appears in the numerator of the right-hand side of this equation should be replaced by $\left(F_{1}+I_{a}-J\right)^{1 / 2}$.

${ }^{26} \mathrm{~J}$. Bardeen and C. H. Townes, Phys. Rev. 73, 97 (1948).
$48 \times 48$ square matrix in which the only nonzero offdiagonal elements are those satisfying the selection rule $\Delta F_{1}= \pm 1$. Furthermore, all matrix elements are independent of $M_{F}$. With these simplifications, solving the secular determinant for the entire $48 \times 48$ matrix reduces to the equivalent problem of solving two quadratic equations. The exact expressions for the firstorder, $J=1 \mathrm{hfs}$ energies which result are listed below:

$$
\begin{aligned}
& W\left(\frac{9}{2}, 5\right)=\left\langle\frac{9}{2}, 5|V| \frac{9}{2}, 5\right\rangle \\
& W\left(\frac{9}{2}, 4\right)=\frac{1}{2}(B+C)+\frac{1}{2}(B-C)\left\{1+[2 \alpha /(B-C)]^{2}\right\}^{1 / 2} \\
& W\left(\frac{7}{2}, 4\right)=\frac{1}{2}(B+C)-\frac{1}{2}(B-C)\left\{1+[2 \alpha /(B-C)]^{2}\right\}^{1 / 2} \\
& W\left(\frac{7}{2}, 3\right)=\frac{1}{2}(D+E)+\frac{1}{2}(D-E)\left\{1+[2 \delta /(D-E)]^{2}\right\}^{1 / 2} \\
& W\left(\frac{5}{2}, 3\right)=\frac{1}{2}(D+E)-\frac{1}{2}(D-E)\left\{1+[2 \delta /(D-E)]^{2}\right\}^{1 / 2} \\
& W\left(\frac{5}{2}, 2\right)=\left\langle\frac{5}{2}, 2|V| \frac{5}{2}, 2\right\rangle
\end{aligned}
$$

where

$$
\begin{aligned}
& B=\left\langle\frac{9}{2}, 4|V| \frac{9}{2}, 4\right\rangle, \\
& C=\left\langle\frac{7}{2}, 4|V| \frac{7}{2}, 4\right\rangle, \\
& D=\left\langle\frac{7}{2}, 3|V| \frac{7}{2}, 3\right\rangle, \\
& E=\left\langle\frac{5}{2}, 3|V| \frac{5}{2}, 3\right\rangle, \\
& \alpha=\left\langle\frac{7}{2}, 4|V| \frac{9}{2}, 4\right\rangle=\left\langle\frac{9}{2}, 4|V| \frac{7}{2}, 4\right\rangle, \\
& \delta=\left\langle\frac{5}{2}, 3|V| \frac{7}{2}, 3\right\rangle=\left\langle\frac{7}{2}, 3|V| \frac{5}{2}, 3\right\rangle .
\end{aligned}
$$

If the off-diagonal elements of $V$ are small compared to the differences between the diagonal elements (such as $B-C$ ) as is the case for $\mathrm{CsF}$, the square roots can be expanded and only the first two terms retained; for the $\left(\frac{9}{2}, 4\right)$ level, for example, $W\left(\frac{9}{2}, 4\right) \cong B+$ $\alpha^{2} /(B-C)$. 